\title{
Use of L-Arginine for Inhaled Nitric Oxide (iNO) Dependent Hepatopulmonary Syndrome (HPS) Post-Liver Transplant
}

\author{
Mohnish Kataria ${ }^{1}$, Abhinav Rastogi' ${ }^{2}$, Sajaad Manjoor ${ }^{3}$, Himanshu Tanwar ${ }^{4}$, Sivaji Patibandla ${ }^{5}$, Vishal V. Singh ${ }^{6}$ \\ 1Department of Gastroenterology, MM Institute of Medical Sciences (Deemed to be University), Mullana, Ambala, \\ Haryana, India. ${ }^{2}$ Department of Cardiology, MM Institute of Medical Sciences (Deemed to be University), Mullana, \\ Ambala, Haryana, India. ${ }^{3}$ Department of Interventional Cardiology, MM Institute of Medical Sciences (Deemed to \\ be University), Mullana, Ambala, Haryana, India. ${ }^{4}$ Department of Cardiology, MM Institute of Medical Sciences \\ (Deemed to be University), Mullana, Ambala, Haryana, India. ${ }^{5}$ Department of Cardiology, MM Institute of Medical \\ Sciences (Deemed to be University), Mullana, Ambala, Haryana, India. ${ }^{6}$ Department of Cardiology, MM Institute of \\ Medical Sciences (Deemed to be University), Mullana, Ambala, Haryana, India.
}

\section{INTRODUCTION}

Hepatopulmonary syndrome (HPS) is one of the recognised complications of liver cirrhosis which warrants urgent liver transplantation. The symptoms usually improve post transplantation but might take 6-12 months to resolve completely. Severe hypoxemia post liver transplantation, especially when done for hepatopulmonary syndrome has been reported. iNO use for the same has been reported in literature with good response. However, it is practically very difficult to continue iNO in a stable child on a portable ventilator due to problems of scavenging.

We report a case of 7-year-old girl who underwent orthotopic liver transplantation (OLT) for severe HPS. Post transplantation the patient had a stormy respiratory course and became severely hypoxemic. She was started on inhaled NO and showed a good response. However, the patient then became NO dependent and multiple attempts to wean iNO failed albeit minimal ventilatory requirements. Following IV L-Arginine infusion, iNO could be weaned within 6 hours without recurrence of hypoxemia. L-Arginine infusion should be considered as a treatment option when facing difficulties to wean iNO in an otherwise well responding patient.

\section{PRESENTATION OF CASE}

A 7-year-old girl was referred to our centre for liver transplantation for hepatopulmonary syndrome secondary to cryptogenic liver cirrhosis. On admission she was centrally cyanosed with oxygen saturations of $75-80 \%$. She was otherwise well and comfortable. She had bilateral grade III digital clubbing. She was noted to have hepatosplenomegaly. The contrast enhanced echocardiography prior to transplant showed a structurally normal heart with multiple aortopulmonary collaterals secondary to pulmonary arteriovenous malformations (AVMs) as concluded by the appearance of microbubbles as contrast in the left atrium within 3 cardiac cycles; there was no evidence of pulmonary hypertension. Liver MRI prior to transplant showed patent portal venous structures and no features of portal hypertension. No other vascular shunts were identified. The patient was urgently listed for liver transplantation, and underwent cadaveric liver transplant. The procedure itself was uneventful. Her ventilation was gradually weaned aiming to keep oxygen saturations $>75 \%$.
Corresponding Author: Dr. Abhinav Rastogi, Department of Cardiology, MMIMSR, Mullana, Ambala, Haryana, India.

E-mail: dr.abhinav2017@gmail.com

DOI: $10.14260 /$ jemds/2020/242

Financial or Other Competing Interests: None.

How to Cite This Article:

Kataria M, Rastogi A, Manjoor S, et al. Use of L-arginine for inhaled nitric oxide (iNO) dependent hepatopulmonary syndrome (HPS) post-liver transplant. J. Evolution Med. Dent. Sci. 2020;9(13):1119-1121, DOI: 10.14260/jemds/2020/242

Submission 22-12-2019, Peer Review 11-03-2020 Acceptance 17-03-2020, Published 30-03-2020.

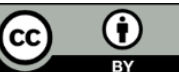


She was extubated on day 3 post OLT to high flow nasal cannula device. She had a postoperative transthoracic echocardiogram which showed good cardiac function with no evidence of significant pulmonary hypertension. On day 4 post OLT she developed a significant pleural effusion on right side which was drained. Day 6 post OLT the patient was changed over to Continuous Positive Airway Pressure Ventilation (noninvasive CPAP) in view of increased work of breathing, falling oxygen saturations, and persistent right lower lobe atelectasis. Her mixed venous saturations then were $35-50 \%$. On the $7^{\text {th }}$ post-transplant day, she had to be intubated and ventilated because of respiratory failure. She was extremely difficult to oxygenate, she required moderate ventilator pressures to achieve acceptable tidal volume. She remained in $100 \%$ oxygen with arterial saturations of $50 \%$. The patient was started on inhaled nitric oxide (iNO) at 5 ppm which improved oxygenation to $70-80 \%$. No difference was noted in oxygenation on high dose inhaled nitric oxide (iNO) of $20 \mathrm{ppm}$. Oxygenation index remained borderline around 40 .

Her ventilation remained stable but multiple attempts to wean iNO were unsuccessful. She eventually underwent tracheostomy to facilitate long term ventilation on day 30 post OLT. She was started on Sildenafil (dose range $0.5 \mathrm{mg} / \mathrm{Kg}$ to 1 $\mathrm{mg} / \mathrm{Kg}$ every 4-6 hourly was tried) to wean iNO (from $5 \mathrm{ppm}$ to $1 \mathrm{ppm}$ ). However, by Day-60, the patient was still dependent on iNO though the ventilator requirements were minimal. Patient was completely interactive but would rapidly desaturate and become cyanosed on stopping iNO(1 ppm). Due to problems in scavenging of iNO with portable ventilators and practicalities in discharging a patient home on iNO, we were desperately looking for an alternative to iNO. On day 68 post-surgery after discussion with regional pulmonary hypertension team patient was started on L Arginine infusion to be able to wean off iNO, (details in the table). Her L-Arginine levels were $32 \mu \mathrm{mol} / \mathrm{L}$ (normal range 26 - 180) prior to commencing L-Arginine.

\section{Weaning Regimen}

Acute-

Intravenous L-Arginine infusion $15 \mathrm{mg} / \mathrm{Kg} / \mathrm{min}$ given over 20 minutes. Sildenafil in the dose of $0.3 \mathrm{mg} / \mathrm{Kg}$ was administered after 2 hours of starting infusion. We weaned FiO2 to 0.3 after 90 minutes of Sildenafil administration. We then increased FiO2 to 0.6 \& stopped inhaled Nitric Oxide after 24 hours of starting L Arginine infusion. Same dose L-Arginine $(15 \mathrm{mg} / \mathrm{Kg}$ ) was given daily intravenously for 1 month

\section{Long Term-}

Arginine was weaned to half the dose every alternate day for 1 month and then dose was gradually tapered over 1 month and stopped. Patient was discharged to local hospital on day 94 post OLT ( 1 month after starting L-Arginine and stopping iNO)on BIPAP through tracheostomy with a plan to wean Sildenafil and L Arginine over 2 months. Her Oxygen saturations were $75-80 \%$. She was in $40 \%$ Oxygen. Patient's blood Arginine level was within normal limits [69 $\mu \mathrm{mol} / \mathrm{L}$ (Range 26 -180)] prior to discharge. Follow-up of this patient 6 months after transplantation revealed that she was discharged home on 0.5 litres/minute of nasal cannula oxygen, tracheostomy has been successfully decannulated and LArginine and sildenafil were stopped as planned. She was reviewed after 2 years for elective abdominal closure. She was noticed to be saturating more than $95 \%$ in room air.

\section{DISCUSSION}

Hypoxemia in patients with liver cirrhosis may occur in the context of hepatopulmonary syndrome (HPS) together with portopulmonary hypertension (PPH). Although portopulmonary hypertension and the hepatopulmonary syndrome are associated with the same underlying diseases, they have contrasting pathophysiological backgrounds, and hence their management is different.

HPS is triad of Hypoxemia, underlying chronic liver disease and intrapulmonary vascular dilations (IPVD). ${ }^{1}$ In the paediatric population the prevalence of HPS is described as 8 $20 \%$ in children with liver cirrhosis. ${ }^{2}$ From a radiological standpoint, HPS is characterized by diffuse dilatation of the peripheral pulmonary vasculature. In this case, the diagnosis of HPS was discussed following contrast-enhanced transthoracic echocardiography with agitated saline in detecting the presence of pulmonary arteriovenous malformations (AVMs). During this procedure, saline is shaken to produce microbubbles greater than $10 \mu \mathrm{m}$ in diameter and administered through a peripheral vein in the arm. The presence of microbubble opacification in the left atrium within 3 to 6 cardiac cycles following opacification of the right atrium is considered a positive test for the presence of intrapulmonary vascular shunting. In consequence, contrast echocardiography is the recommended study for the evaluation of IPVD in diagnosing HPS, as it is more sensitive than the lung perfusion scan with technetium and has no radiation exposure. CT angiogram showed the findings of dilated pulmonary vasculature (figure 1). Thus, CT angiogram can be helpful in a patient with liver disease and unexplained hypoxemia.

Mortality of HPS has been reported to be around $25 \%$ to $46 \% .^{3}$ This syndrome is due to abnormal intrapulmonary vascular dilatation, which results in an excess perfusion for a given state of ventilation. This complication is characterised by anatomical shunting and a diffusion-perfusion abnormality. ${ }^{4}$ Evidence is growing rapidly that excess production of Nitric Oxide plays a central part in pathogenesis of HPS. ${ }^{5}$ Excess production of Nitric oxide is secondary to altered bowel perfusion and an increased rate of enteral translocation of gram-negative bacteria and endotoxin, due to altered bowel perfusion. ${ }^{6}$ Diets containing low amount of Arginine (substrate for nitric oxide),IV Methylene blue have been tried without much success. Complete resolution of condition posttransplant is well documented.

In the literature, cases of transient or fatal deterioration of intrapulmonary shunting post OLT with dramatically worsening hypoxia had been described. Schiller et al reported a case of 10 year old patient who was treated with inhaled Nitric oxide for post OLT hypoxemia. iNO was stopped successfully on day 14 post OLT in their patient. ${ }^{7}$ In another case report by PH Durand at al, patient responded very well to NO and they were able to wean NO by day 10 post OLT. ${ }^{8}$

In our patients multiple attempts to wean and stop NO were unsuccessful and patient would become clinically cyanotic with oxygen saturations $<60 \%$ if iNO was dropped to less than $<1 \mathrm{ppm}$. Patient was essentially iNO dependent for 8 
weeks post OLT. Clearly the respiratory process in our patients was iNO responsive. The alternative therapies to iNO include IV L Arginine infusion, IV Citrulline infusion.
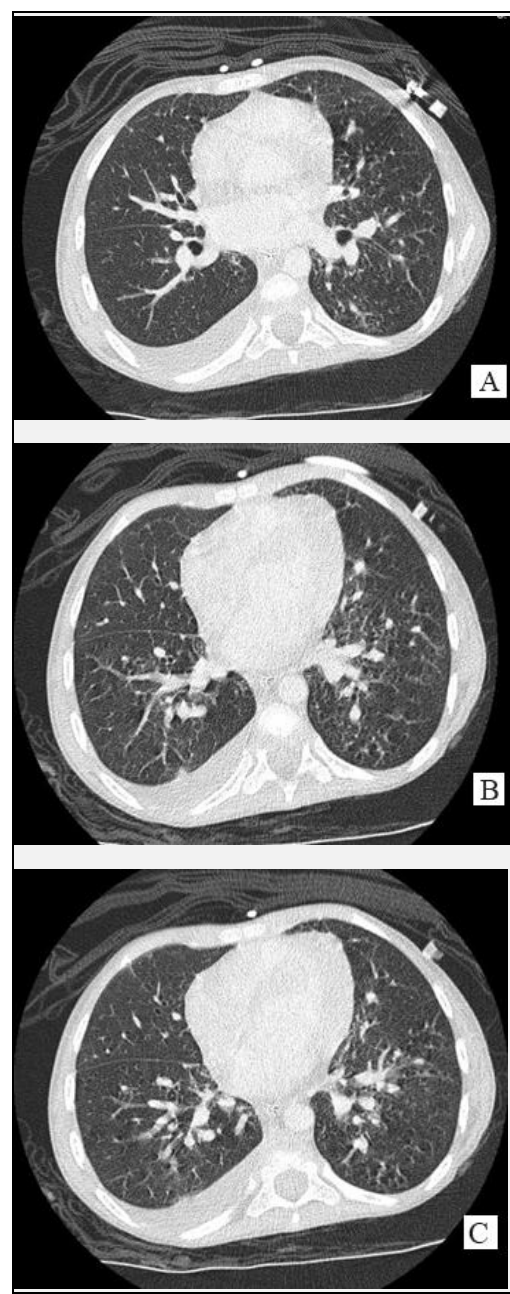

Figure 1. Axial Computed Tomography Angiography Images Using Intravenous Contrast below the Level of Carina (A) Towards the Base $(B, C)$. Diffuse Dilatation of the Pulmonary Vasculature is Seen to be More Prominent in Caudal Sections $(B, C)$. Lung Parenchyma is Normal

L-Arginine is the nitrogen donor for synthesis of nitric oxide. Hence in theory administration of L Arginine would increase endogenous production of Nitric oxide which is a potent pulmonary arterial vasodilator. Several studies have demonstrated therapeutic benefits of L-Arginine therapy for pulmonary hypertension. ${ }^{9}$ L-Arginine infusion has decreased pulmonary vascular resistance and improved oxygenation in infants with persistent pulmonary hypertension. ${ }^{10}$ L-Arginine supplementation has been shown to improve pulmonary artery pressures and haemodynamics in patients with primary and secondary pulmonary hypertension ${ }^{9}$. Claudia et la, demonstrated that Oral Arginine produced a $15.2 \%$ mean reduction in estimated pulmonary artery systolic pressure after 5 days of therapy in 10 patients with sickle cell disease. Due to problems in using iNO on a portable ventilator associated with problems in use of iNO at home, we were desperate to find an alternative to iNO which in our case was L-Arginine. The protocol we followed was based on a study protocol used by Schulze-Neick at al. in their study on children with cardiac disease. ${ }^{11}$ It was modified to suit our patient's needs.

In all previous case reports of hypoxemia post OLT, response to iNO was good and weaning of iNO was possible within 2 weeks post OLT without recurrence of hypoxemia. To the best of our knowledge this is a first case reported in literature where in patient who underwent OLT for HPS became hypoxic post-surgery and remained iNO dependent, but responded well to $\mathrm{L}$ Arginine infusion. We therefore recommend use of L-Arginine and low dose Sildenafil in case of iNO dependent patient with HPS.

\section{REFERENCES}

[1] Krowka MJ, Cortese DA. Hepatopulmonary syndrome. Chest 1990;98(5):1053-4.

[2] Noli K, Solomon M, Golding F, et al. Prevalence of hepatopulmonary syndrome in children. Pediatrics 2008;121(3):e522-7.

[3] Barbe T, Losay J, Grimon G, et al. Pulmonary arteriovenous shunting in children with liver disease. J Pediatr 1995;126(4):571-9.

[4] Whyte MK, Hughes JM, Peters AM, et al. Analysis of intrapulmonary right to left shunt in the hepatopulmonary syndrome. J Hepatol 1998;29(1):8593.

[5] Vallance P, Moncada S. Hyperdynamic circulation in cirrhosis: a role for nitric oxide? Lancet 1991;337(8744):776-8.

[6] Nunes H, Lebrec D, Mazmanian M, et al. Role of nitric oxide in hepatopulmonary syndrome in cirrhotic rats. Am J Respir Crit Care Med 2001;164(5):879-85.

[7] Schiller O, Avitzur Y, Kadmon G, et al. Nitric oxide for postliver-transplantation hypoxemia in pediatric hepatopulmonary syndrome: case report and review. Pediatr Transplant 2011;15(7):E130-4.

[8] Durand P, Baujard C, Grosse AL, et al. Reversal of hypoxemia by inhaled nitric oxide in children with severe hepatopulmonary syndrome, type 1, during and after liver transplantation. Transplantation 1998;65(3):437-9.

[9] Mehta S, Stewart D, Langleben D, et al. Short-term pulmonary vasodilation with L-arginine in pulmonary hypertension. Circulation 1995;92(6):1539-45.

[10] McCaffrey M, Bose CL, Reiter P, et al. Effect of L-arginine infusion on infants with persistent pulmonary hypertension of the new-born. Biol Neonate 1995;67(4):240-3.

[11] Schulze-Neick I, Penny DJ, Rigby ML, et al. L-arginine and substance $P$ reverse the pulmonary endothelial dysfunction caused by congenital heart surgery. Circulation 1999;100(7):749-55. 